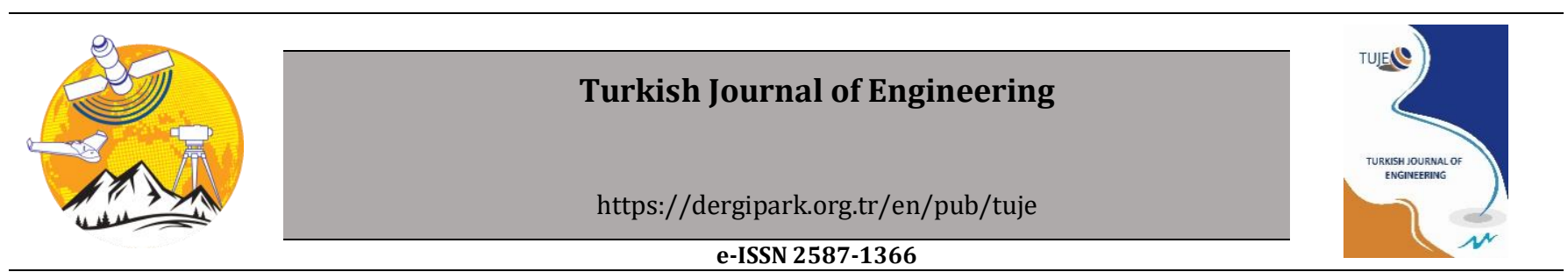

\title{
Classification of UAV point clouds by random forest machine learning algorithm
}

\author{
Mustafa Zeybek ${ }^{* 1}$ (D) \\ ${ }^{1}$ Artvin Coruh University, Engineering Faculty, Geomatics Engineering Department, Artvin, Turkey
}

\author{
Keywords \\ Unmanned aerial vehicle \\ Point cloud \\ Classification \\ Random forest
}

\begin{abstract}
Today, unmanned aerial vehicle (UAV)-based images have become an important data sources for researchers who deals with mapping from various disciplines on photogrammetry and remote sensing. Reconstruction of an area with three-dimensional (3D) point clouds from UAV-based images are an essential process to be used for traditional 2D cadastral maps or to produce a topographic maps. Point clouds should be classified since they subjected to various analyses for extraction for further information from direct point cloud data. Due to the high density of point clouds, data processing and gathering information makes the classification of point clouds a challenging task and may take a long time. Therefore, the classification processing allows an optimal solution to acquire valuable information. In this study, random forest machine learning algorithm for classification processing is applied with radiometric features (Red band, Green band and Blue band) and geometric characteristics derived from covariance feature (curvature, omnivariance, flatness, linearity, surface variance, anisotropy and normalized terrain surface) of points. In addition, the case study is presented in order to test applicability of the proposed methodology to acquire an accuracy and performance of random forest method on the UAV based point cloud. After the classification processing, a class assigned each point from the model was compared with the reference data class. Lastly, the overall accuracy of the classification was achieved as $96 \%$ and the Kappa index was reached to $91 \%$ on data set.
\end{abstract}

\section{INTRODUCTION}

Three-dimensional (3D) high spatial accuracy point cloud has become an important data source for various disciplines such as urban planning, simulation, mapping, visualization, and emergency response. Satellite-based methods, aerial camera and laser systems and terrestrial methods are used in the production of these models (Pandey et al. 2019). The area size is very important criteria. Larger area requires a greater number of quantified persons, which leads more cost and labour force.

Difficulties arise in the production of traditional 2D urban maps from unmanned aerial vehicle (UAV) data, especially due to the existence of complex structures. So, it is difficult to obtain information from 3D dense point cloud data. One of the basic processing in solving these problems is classification of point clouds (Özbay and Çınar 2016; Zeybek and Şanlıoğlu 2019a).

Over the last decade UAV map production systems have become an important technology for different disciplines (Akgül et al. 2016; Öztürk et al. 2017; Ulvi 2018; Ulvi and Toprak 2016; Ulvi et al. 2020). The main reason for the spread of this system is the availability of flight platforms and technology for civilian users, as well as reductions in costs. Moreover, the high resolution and density of the data allow to use the data in analyses that can be adapted for different purposes for researchers and civilian users. Besides easy access to 3D data, problems have arisen in processing intensive data and to extract necessary information (Karakaş 2018). The classification procedures play a vital role for solving these problem (Zeybek and Şanlığlu 2019a).

In addition to UAV systems, airborne (ALS) and terrestrial (TLS) based LiDAR applications are widely for mapping purposes used (Sevgen 2019; Demir 2015). Laser scanning devices, global navigation satellite system (GNSS) and inertial measurement unit (IMU) sensors are synchronized systems that integrated on the moving (MLS) platforms, such as aircraft, helicopters and satellites. Point cloud densities vary according to the altitude and flight speed of the platform to the ground 
surface. However, acquired data still requires classification processing for map production.

In general, each point in the point clouds obtained with a LiDAR system contain several information such as incidence angle, slope distance, and intensity values (Shan and Toth 2018). Laser signal intensity has been used in various applications such as classification of natural and built materials, urban pavement surfaces, determination of snow covered areas, soil and rock properties, coastal, land cover and flood modelling, road and road marking extractions (Kashani et al. 2015). The surface type can be recognised using the reflected laser signal intensity, i.e. the measure of the return signal strength (Yadav and Singh 2017). On the other hand, a considerable research group worked on the development of various filtering methods for classification of LiDAR point clouds such as interpolation-based (Kraus and Pfeifer 1998), slopebased (Vosselman 2000), segmentation-based (Tóvári and Pfeifer 2005) and morphological (Zhang et al. 2003).

Multispectral LIDAR, which is a new remote sensing technology is able to measure both spectral and spatial information simultaneously and has proven to be usable method in various fields today (Chen et al. 2017; de Almeida et al. 2019; Wei et al. 2012; Guyot et al. 2019; Luo et al. 2019; Niu et al. 2015; Pan et al. 2018). The first commercial aerial multispectral LiDAR, Optech Titan (532, 1,064 and 1,550 nm), has been brought new advantageous to the multispectral LiDAR land cover classification process (Wichmann et al. 2015).

It is stated that the filtering methods produce effective results bot only for LIDAR data classification but also UAV data classification (Zeybek and Şanlıoğlu 2019a). In addition, new approaches and methods have been proposed constantly (Şahin et al. 2018). It is possible to classify point clouds by utilizing of the geometrical properties as well as multispectral band properties. Machine learning and deep learning algorithms have been also used to classification of dense point cloud data (Özdemir and Remondino 2019).

Depending on the application, supervised and unsupervised classification approaches are proposed (Zou et al. 2017), and all approaches are based on descriptive characteristics. Some well-known image processing techniques were applied for classification purposes by examining the geometric properties on the 3D point cloud data (Zhang et al. 2003).

The Markov random field (MRF) classifier was used for power line and building classification using the local linear and planar characteristics of points in city model data acquired from ALS for classification (Sohn et al. 2012).

In some paper, multi-source optical image and LiDAR data features for urban scene classification has been studied (Guo et al. 2011). Hyperspectral imaging in individual tree detection with UAV based classifications were also investigated (Nevalainen et al. 2017). Niemeyer et al. (2014) proposed a context-based conditional random forest classification method for urban LiDAR point clouds. Çömert et al. (2019) studied the performance of the random forests algorithm for mapping of burned forest areas from remotely sensed images.

In this article, Random Forest (RF) classification algorithm on $3 \mathrm{D}$ dense UAV point clouds have been investigated for classification feasibility and accuracy for urban area.

\section{MATERIAL AND METHOD}

The proposed methodology consists of three steps; automatic classification of each point with the machine learning algorithm $\mathrm{RF}$, utilizing radiometric and geometric properties for point clouds. The general workflow of the proposed methodology is given in Fig 1.

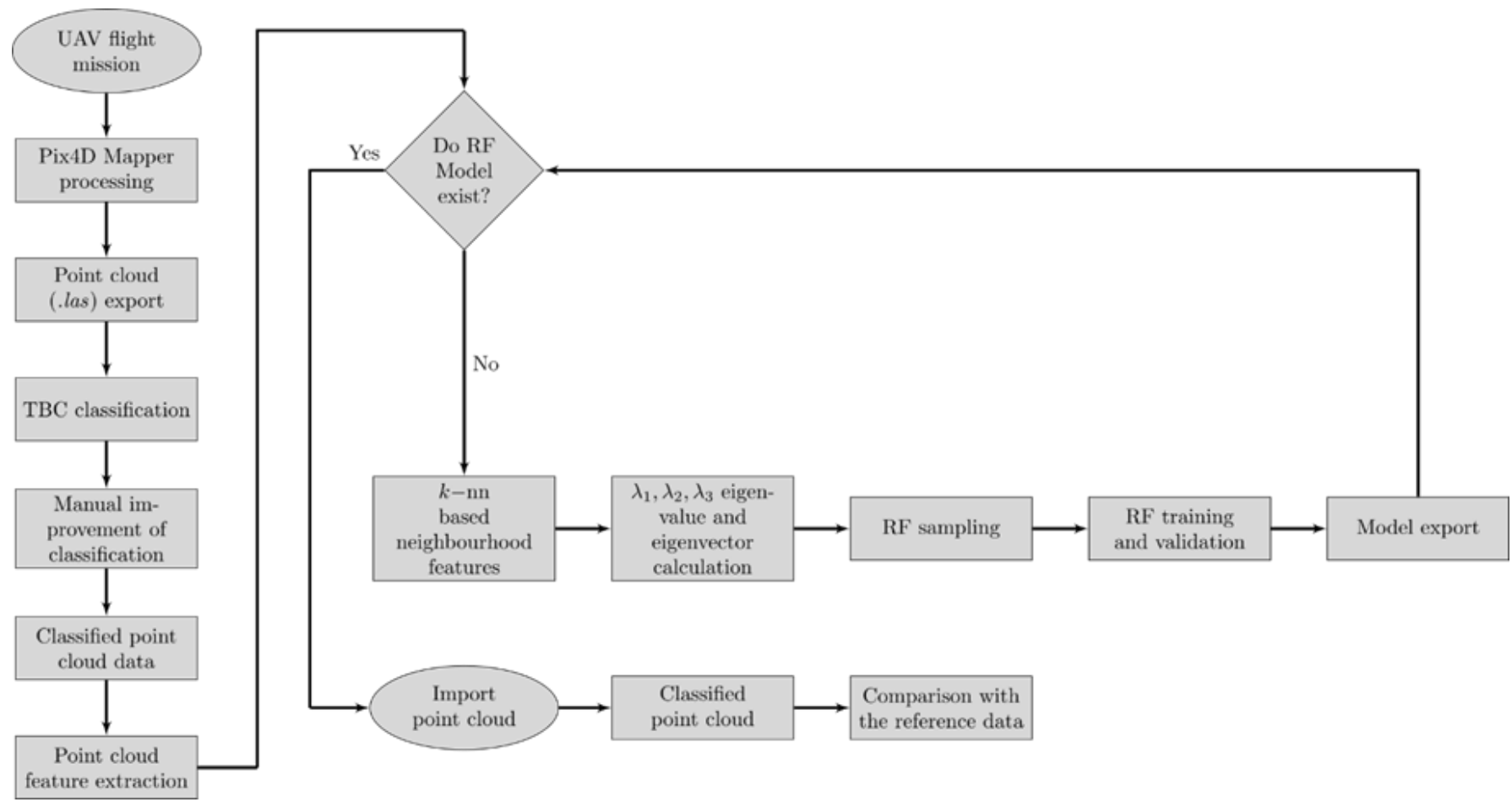

Figure 1. General work flow chart 


\subsection{Study Area}

Artvin Coruh University Seyitler Campus has been selected as the study area. The total surveyed area is 87.2641 ha. The boundaries of the study area are in the range of latitude $(41.8440,41.8560)$ and longitude (41.1940, 41.2100). The average ellipsoidal height (GRS80) value is around $567 \mathrm{~m}$. Details of the study area are given in Figure 1.

Several major buildings (Engineering, and Vocational school and Rectorate) and President's Office are located in the study are. The general topographic structure of Artvin is rugged. However, the study area has a flat topographic feature.

\subsection{UAV Point Cloud}

2D images were acquired from the UAV platform to produce the UAV point cloud.

UAV platform used in this study was Phantom 4 RTK brand and model 4 motorized. Thanks to the real-time kinematics (RTK) system, which is one of the main features of the UAV platform, images are obtained directly in georeferenced. In the EXIF information, global navigation satellite system (GNSS) information is integrated in detail. Therefore, ground control point (GCP) is not mandatory or third-party data processing software is not required for georeferencing purposes.

PIX4DMapper software was used in this study, which is a commercial software and commonly used for image processing. (https://www.pix4d.com/). This software package with an easy-to-use interface consists of two stages to produce dense point cloud. The first stage is a keypoint image feature extraction process which is similar to scale invariant feature transform (SIFT) (Lowe 2004). Algorithm identifies the specific points in each image with similar structure for allowing the images to match image pairs corresponding these keypoints (Alignment) (Zeybek and Şanlığlu 2019b). After the image matching stage, the camera calibration optimization step is performed to update the camera parameters to improve the estimation of lens distortion parameters with physical lens properties.

The next processing stage is the dense point matching, whereby the point clouds are densified by starting similar location or point matching operations on the images whose camera location and orientation parameters are calculated. In this stage the density of the points are increased which leads accurate representation of the surface. A more dense and accurate surface model is produced when compared to the sparse point clouds.

\subsection{Random Forest Classifier}

Breiman (2001) proposed a new approach, which is called random forest (RF) classification algorithm, by adding a layer of randomness to the bagging method. It is very easy to use since only two parameters the number of variables in the random subgroup of each node and the number of trees in the forest) are required. In addition, the RF classification algorithm is not sensitive to outliers.

It is an ensemble tree-based learning algorithm. The $\mathrm{RF}$ classifier contains set of decision trees in a subset of a randomly selected training set. For decision-making, the final class of the test object is given aggregated votes from different decision trees (Akar and Güngör 2012; Sevgen 2019; Ok et al., 2011). This algorithm is preferred in this study because the classification accuracy is higher than most other decision trees.

The basic working principle of the random forest algorithm is as follows (Ramasubramanian and Singh 2017),

1. Several inputs are required in this algorithm such as number of observations $(\mathrm{N})$, number of decision three (ntree) and number of variables in the dataset (M). Users can select number of decision three (ntree) input then other two inputs are selected automatically by algorithm.

2. A subset of the number of predictors sampled for splitting at each node (mtry) (Kuhn 2008; Liaw and Wiener 2002) variable is selected from M, where mtry $<\mathrm{M}$ and a random set of mtry variables are created in the decision tree,

3. Each tree should be as large as possible,

4. The majority vote is used to assign the class of observation.

To form each decision tree, a randomly selected subset of $\mathrm{N}$ observations is used without any changes (normally 2/3). For more stable models and accurate generation of variable importance estimations, a large number of trees (ntree) is recommended. However, large number of trees (ntree) leads more memory and longer processing times. For small data sets, 50 trees may be sufficient, while larger data sets may require 500 or more. Therefore, parameter tuning can be made in the field using trial and error (Cutler et al. 2007). There is a wide discussion in the literature about the effect of mtry and ntree parameters. It is also reported that models of different mtry values do not affect the correct classification rates and other performance metrics are fixed under different mtry values (Cutler et al. 2007).

The first step for the generation of the RF model is the preparation of training data. In the creation of reference data, which is also known as ground-truth data, manual classification is performed in a specific field. The number of classes should be determined according to the relevant field characteristics. In this study, the relevant area was divided into 3 different classes namely, ground, vegetation and building classes.

The geometric characteristics of the points representing to these classes were calculated with the help of eigenvalues and eigenvectors according to the covariance matrix in k-nearest neighbourhood (k-nn) criteria and values (Blomley et al. 2014; Sevgen 2019; Hackel et al. 2017; Taşcl and Onan 2016).

Ground points at the point cloud were determined using a cloth simulation filtering (CSF) algorithm (Zhang et al. 2016). The digital terrain model (DTM) was created with the ground points. The distance of the non-ground points to the DTM surface was calculated. Thus, the normalized feature, which is also known as above ground level (AGL) is computed.

Calculated geometric and radiometric thirteen different features, which is used to produce the model with the highest accuracy for reference data, have been 
created. Hence, a classifier model is produced which can be adapted to the whole area or different study area.

For the RF model training, 20000-point sample data that including all classes were used for learning stage. The sample data was partitioned as $70 \%$ and $30 \%$ for training and validation stage, respectively.

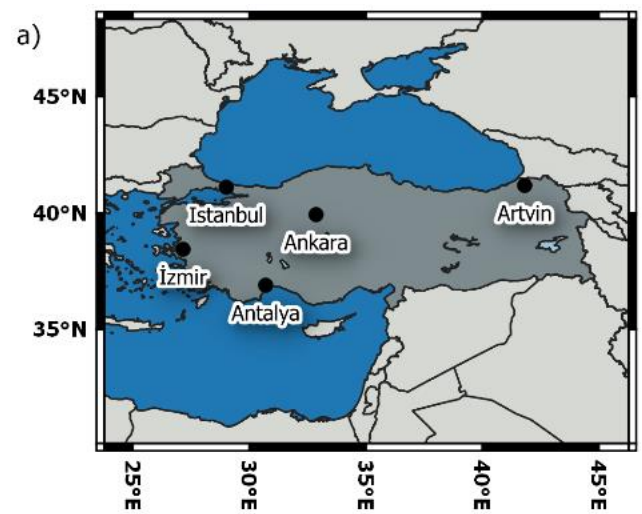

b)

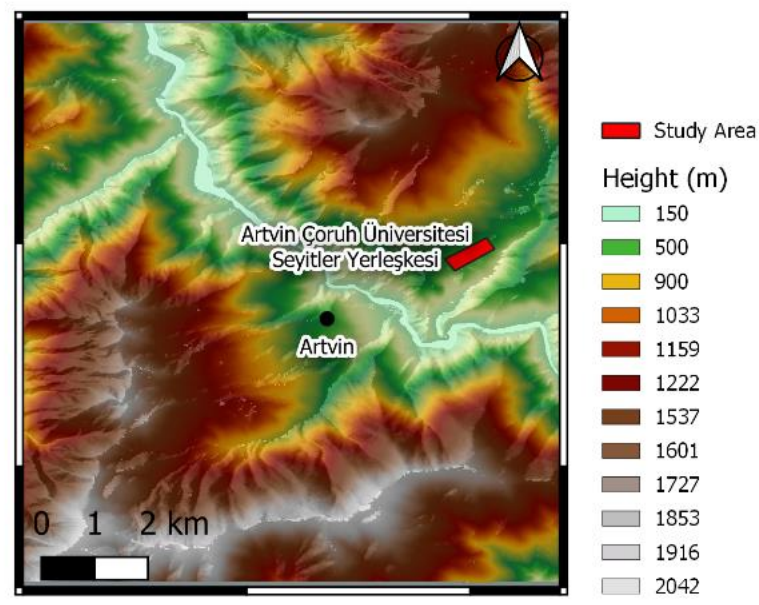

c)

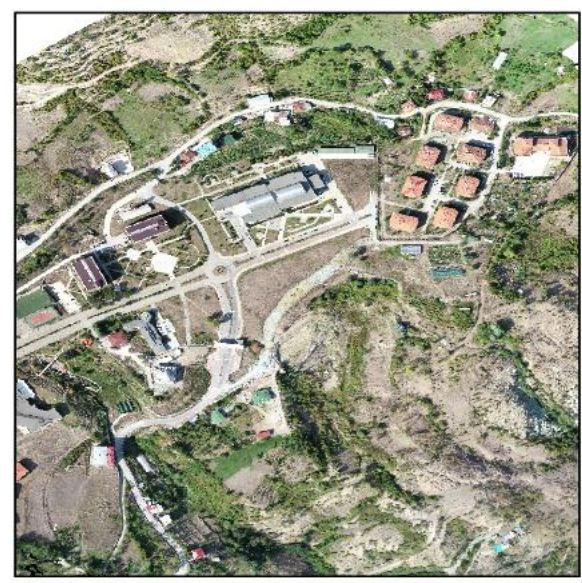

Figure 1. Study area, a) Location of Artvin province, b) Height values of Artvin region, c) Orthomosaic image of the study area

The RF models are various, and the algorithm randomly selects the mtry variables from the set of available estimators when creating each node in a tree. Therefore, while creating a node, a different set of random variables in which is presenting the best separation point is selected. Since there are thirteen variables, thirteen random mtry values are examined. The number of trees (ntree) is commonly considered as
500 in the classification studies. The parameters specified for the RF classifier are left to the user's preference. However, it is known that it does not affect accuracy after certain values (Ok et al. 2011).

\subsection{Accuracy Assessment Criteria}

Accuracy analysis in the classification of point cloud shows the consistency between the reference data class and the model based assigned class of each point. Reference data classification was done with point clouds classification module using the Trimble Business Center (TBC) commercial software. After the reference classification, cross-sectional analyses were performed with the help of the operator, and improvements were obtained in the classification. Thus, the comparison with the model classification was made more accurately.

Accuracy assessment procedure is performed to determine the classification accuracy of the model produced by RF based on the reference data. The process is the statistical evaluation of each point in the point cloud after the RF model classification by comparing it with reference class.

The commonly used statistical methods in accuracy evaluations are relative values obtained according to error matrix (confusion matrix, contingency table) (Çetinkaya and Toz 2007). Error matrices show the correlation between reference data from binary or more classes and model classification results (Table 1 ).

Table 1. Error matrix

\begin{tabular}{c|lll}
\hline \multirow{2}{*}{} & & \multicolumn{2}{c}{ Reference } \\
\cline { 3 - 4 } & & Class 1 & Class 2 \\
\hline \multirow{2}{*}{$\bar{D}_{\Sigma}$} & Class 1 & A & B \\
\cline { 2 - 4 } & Class 2 & C & D \\
\hline
\end{tabular}

The quality of classification accuracy is based on the values calculated from the error matrix (Table 1) and the criteria given in Eq. (1)-(11).

\subsection{Implementation}

Pix4DMapper commercial software was used to convert UAV images to point clouds. Then, Open-source CloudCompare (CloudCompare 2013) for visualization, R Programming language (Team 2019) for RF application and commercial TBC software were used for analysis on point clouds. A prototype framework was developed for the proposed RF-based point cloud classification method using R programming language.

\section{RESULTS AND DISCUSSIONS}

Totally 142 images were taken in the study area and 23155722 three-dimensional point data were generated (Figure 2). The flight height was approximately $200 \mathrm{~m}$ which was suitable with the topographic rugged terrain and the ground sampling interval was obtained as 5.93 $\mathrm{cm}$.

RTK system on the platform was connected to Continuously Operating Reference Station (Cors-TR) network. Thus, the images were directly geolocated 
without using any ground control point. The root mean square errors for X, Y, Z coordinates were $1.3 \mathrm{~cm}, 1.4 \mathrm{~cm}$ and $1.4 \mathrm{~cm}$, respectively. The GNSS positional errors for the camera locations are given in Figure 2 and the distributions in Figure 3. The generated dense point cloud was georeferenced in the TUREF / TM42 (EPSG:5258) coordinate system. The point density was obtained as 12 points $/ \mathrm{m}^{3}$. This value can be increased in
SfM software. However, in general, the density values where obtained in this study provide to draw traditional map with accuracy standards. The distance between the points is set at $10 \mathrm{~cm}$ in order to provide pattern of the point cloud and improve the data processing performance. As a result, 20294012-point data was processed.

$$
\begin{aligned}
& \text { Sensivity }=A /(A+C) \\
& \text { Specificity }=D /(B+D) \\
& \text { Prevalence }=(A+C) /(A+B+C+D) \\
& P P V=(\text { Sensivity } * \text { Prevalence }) /((\text { Sensivity * Prevalence })+((1-\text { Specificity }) *(1-\text { Prevalence }))) \\
& \text { NPV }=(\text { Specificity * }(1-\text { Prevalence })) /(((1-\text { Sensivity }) * \text { Prevalence })+((\text { Specificity }) *(1-\text { Prevalence }))) \\
& \text { Detection Rate }=A /(A+B+C+D) \\
& \text { Detection Prevalence }=(A+B) /(A+B+C+D) \\
& \text { Balanced Accuracy }=(\text { Sensitivity }+ \text { Specificity }) / 2 \\
& \text { Precision }=A /(A+B) \\
& \text { Recall }=A /(A+C) \\
& \left.\left.F 1=\left(1+\text { beta }^{2}\right) * \text { Precision } * \text { Recall / ((beta }{ }^{2} * \text { Precision }\right)+ \text { Recall }\right)
\end{aligned}
$$
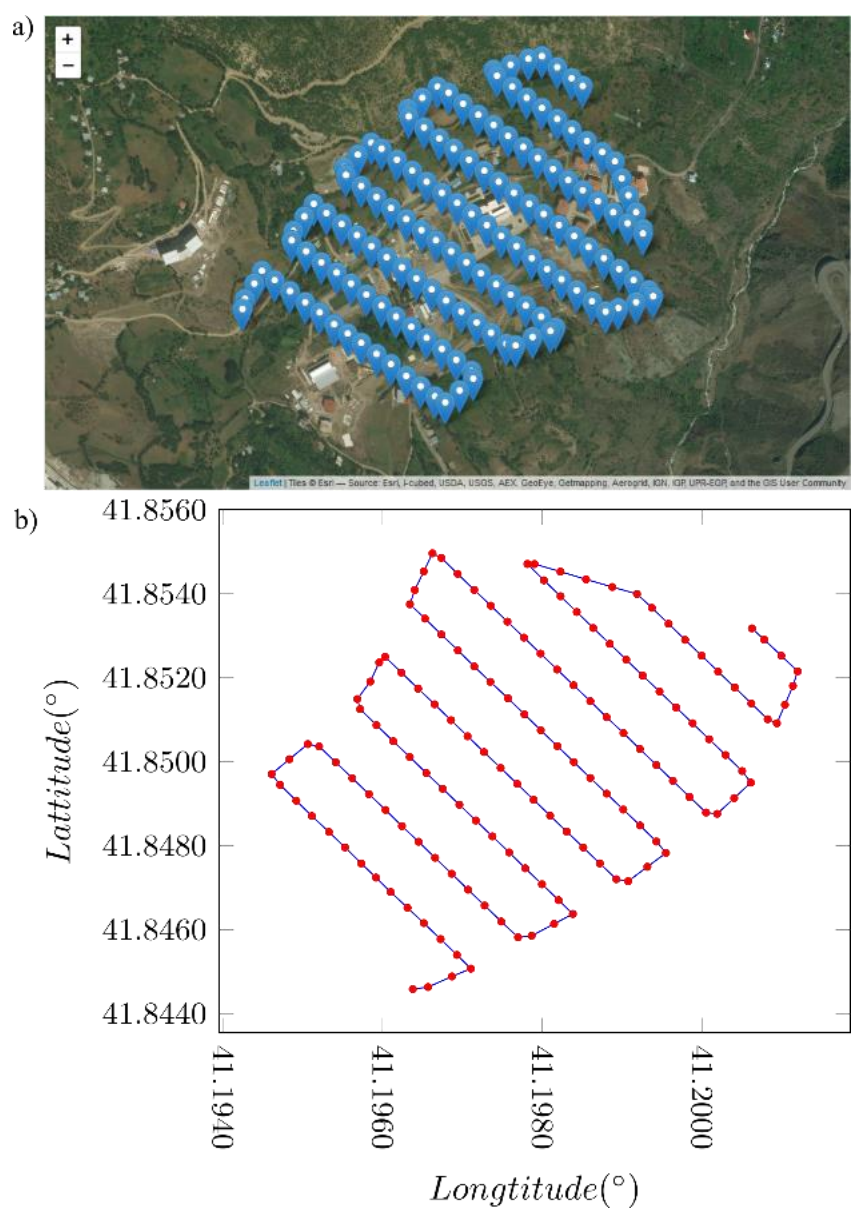

Figure 2. Camera locations according to the GNSS coordinate (WGS 84) in the EXIF data a) camera locations on Leaflet satellite image and b) camera locations on map (red dots: indicating camera locations on WGS-84 coordinate system)
In the study, the test area was determined for the RF model to evaluate the effectiveness of the applied methodology.

Classification of the reference data set was automatically classified using the TBC point cloud module (Figure 4). The TBC uses the default settings and the geometry between points when extracting ground points. This avoids a long trial and error process, especially for the observations involving noisy and outlier points in a point cloud. This feature is effective on rough ground or dense vegetation areas because more than one iteration can eliminate more noisy points. It also saves time for users who are only interested in ground points, such as contour maps production from ground points. It classifies buildings, trees, power transmission lines and poles based on similar geometric features in above ground points. Due to the commercial software, the evaluation of parameters and algorithms related to classification is limited. Exported point clouds were obtained in las format.

According to the American Society for Photogrammetry and Remote Sensing (ASPRS 2019), classification fields are standardized in the point cloud file format las extension as ground points class to 2 , vegetation points class to 5 and building points class to 6 . This file format is designed to contain records of point cloud data from LiDAR or different sensors. Point cloud data is usually converted to this format from software that combines GPS, IMU and laser signal information to generate $\mathrm{X}, \mathrm{Y}$ and $\mathrm{Z}$ point coordinates. The main purpose of the data format is to provide an open format that allows different LiDAR hardware and software tools to input or output data in a common format (ASPRS 2019). 
a)

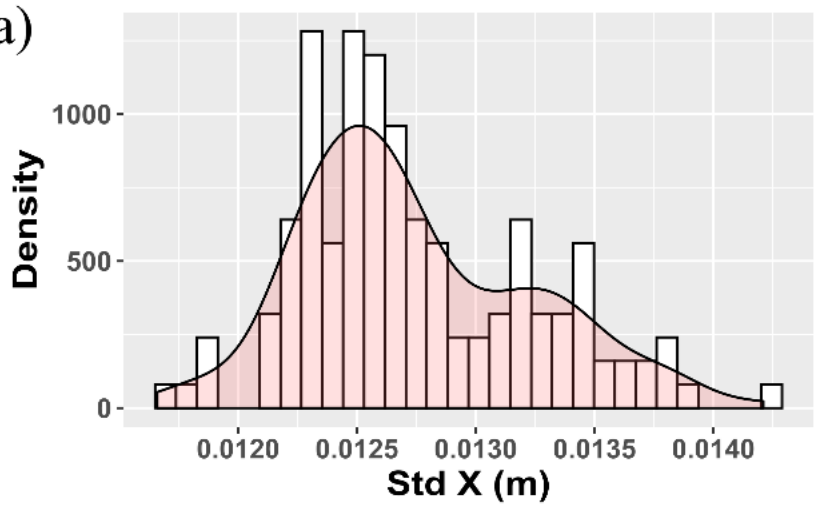

b)

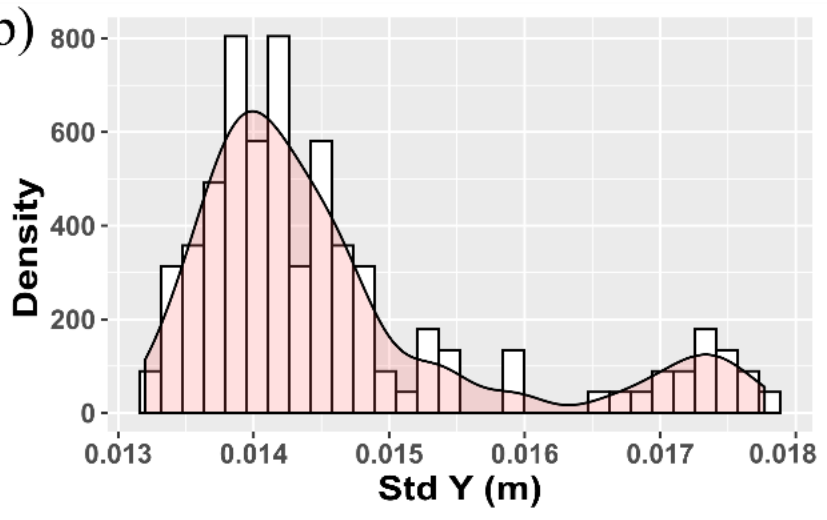

c)

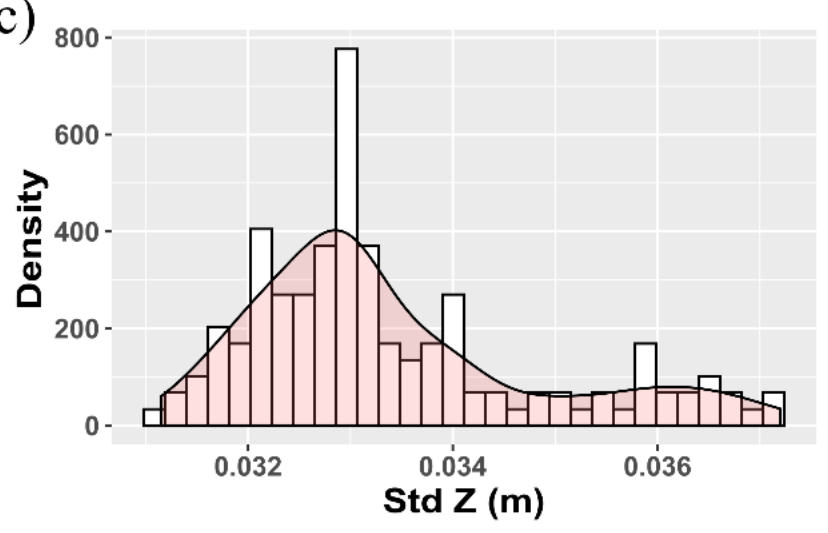

Figure 3. GNSS RMSE's for camera locations a) X-axis direction, b) Y-axis direction and c) Z-axis direction

After automatic classification, classification quality was improved by manual correction. This process was performed again with the help of TBC sectioning at crosssectional intervals of $5 \mathrm{~m}$ (Figure 5).

In the $\mathrm{R}$ programming language, the lidR package was used to read the las file format without corruption. With the readLAS function, the path where the las file is located was determined and the required fields were selected and imported. This function supports the LAS formats 1.1 to 1.4 (Roussel and Auty 2017). An imported file stored in LAS object which inherits from a spatial object from sp package (Bivand et al. 2008). Since there will be applications related to the neighbourhood relations, a certain number of neighbourhood distances must be calculated for each point. For this purpose, considering the dense point clouds, a fast distance calculation should be applied. The nn2 function included in the RANN package has been implemented in this application (Arya et al. 2019).

In the machine learning step, parallel computation has been applied to use the hardware performances at high capacity for intensive training process. The makeCluster function included in the parallel package uses multiple processors to solve problems. Simultaneous training and calculations are divided into different parts. In this way, each part is divided into different command series and the given commands are run on different processors at the same time.

The CreateDataPartition function included in the caret package (Kuhn 2008) is used to create balanced partitions of the training data that will be used to create the model. The class value is evaluated as a factor data type at the result of this function in R. Random sampling takes place within each class, and the overall class distribution of the data is preserved. The RF model is trained with 20000 points obtained from the reference data, which includes all classes for classification.

In this study, a k-fold CV was used to randomly split the training dataset into $\mathrm{k}$ parts, and then each of the $\mathrm{k}$ parts evaluated as a test dataset for the trained model in the other $\mathrm{k}-1$. The average of $\mathrm{k}$ error is calculated for all evaluated parts. Then $\mathrm{k}$-fold $\mathrm{CV}$ was repeated 3 times. In order to determine the model accuracy criteria, the k-fold cross-validation was performed and the $\mathrm{k}$ value was taken as 10. trainControl function was used for this implementation.

The predict function was applied for the estimation of new data of the parameters obtained from the results of $\mathrm{RF}$ model fitting functions. The predict function performs predictions by calling certain methods that depend on the class of the first data object.

Cross tabulation of classes observed and estimated with related statistics was calculated with the confusionMatrix function.
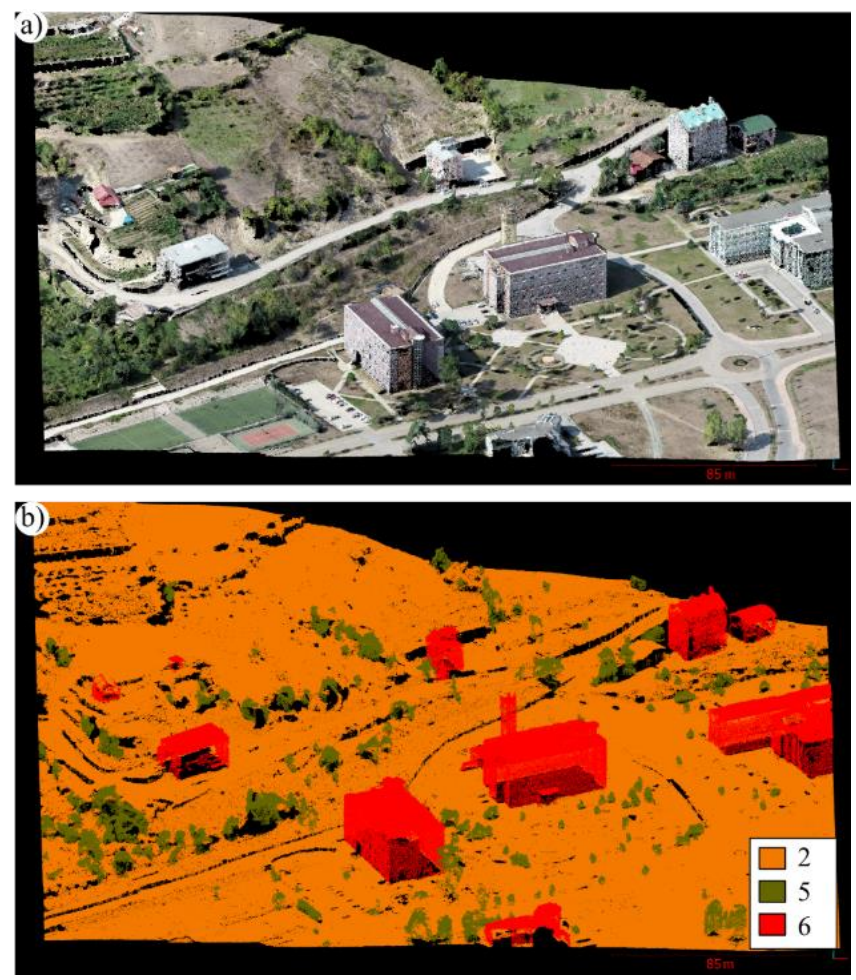

Figure 4. Reference data classification on TBC software a) View of dense point cloud in RGB band values, b) Automatic classified and manually corrected point cloud classes by TBC (ground:2, vegetation:5 and building:6) 

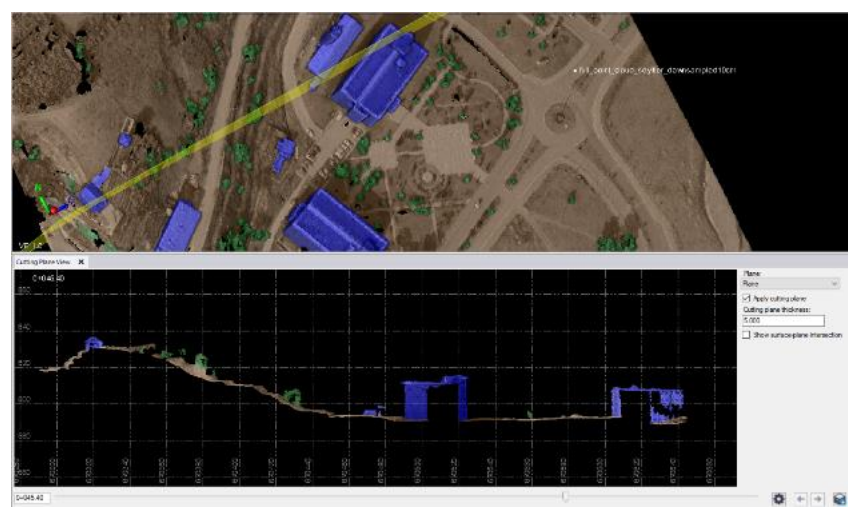

Figure 5. Improvement on the automated classified reference data by means of sections and operator

The resulted accuracy values in the sub-groups were obtained. The obtained maximum accuracy values were calculated for each group. The number of variables available for division in each tree node was randomly calculated for thirteen different variables (Table 2). The mtry value was taken as 10 because it produces the optimum model accuracy at 10. The accuracy of the classification with the various mtry feature variable is differed by approximately $1 \%$. Without the normalizing geometric and radiometric features, RF classifier model produced the $96 \%$ overall accuracy. The most applicable or "true" value for the ntree is not usually very unstable, because it is clear that the predictions from the model are not change much after a certain number of trees.

After the verification process is completed and the accuracy criteria are met, the produced RF model must be stored. The ".rds" file was used to save and load a single R object, which is the RF model, to a link (usually a file) for later use in various data. This process provides to store a more model parameters with specific format than just saving and reading parameter lists. For the produced model, ".rds" file extension is used. saveRDS and readRDS functions are used to save and read the files in ".rds".

Table 2. Model accuracy values for RF model subsets (* mtry subset variable number)

\begin{tabular}{lll}
\hline${ }^{*}$ mtry & Accuracy & Kappa \\
\hline 1 & 0.9500038 & 0.8846614 \\
2 & 0.9537180 & 0.8932820 \\
3 & 0.9570273 & 0.9009979 \\
4 & 0.9593365 & 0.9063616 \\
5 & 0.9607650 & 0.9096831 \\
6 & 0.9614077 & 0.9111628 \\
7 & 0.9623363 & 0.9132596 \\
8 & 0.9632884 & 0.9154454 \\
9 & 0.9634072 & 0.9157523 \\
10 & 0.9635027 & 0.9159197 \\
11 & 0.9631454 & 0.9151173 \\
12 & 0.9629550 & 0.9146240 \\
13 & 0.9625026 & 0.9135558 \\
\hline
\end{tabular}

The method applied to calculate the importance of the variables used for the objects produced by methods specific to train and method was made with the varImp function. When the importance levels of neighbourhoodrelated covariance characteristics were examined, the most important parameter was the above ground level
(AGL) (Figure 7). Radiometric B band and vertical directional surface normal value were also other two parameters that are important classifier features. AGL was the only topographic feature that helps to distinguish between ground and surface classes. Another important feature can be explained to be effective in determining the above-ground points.
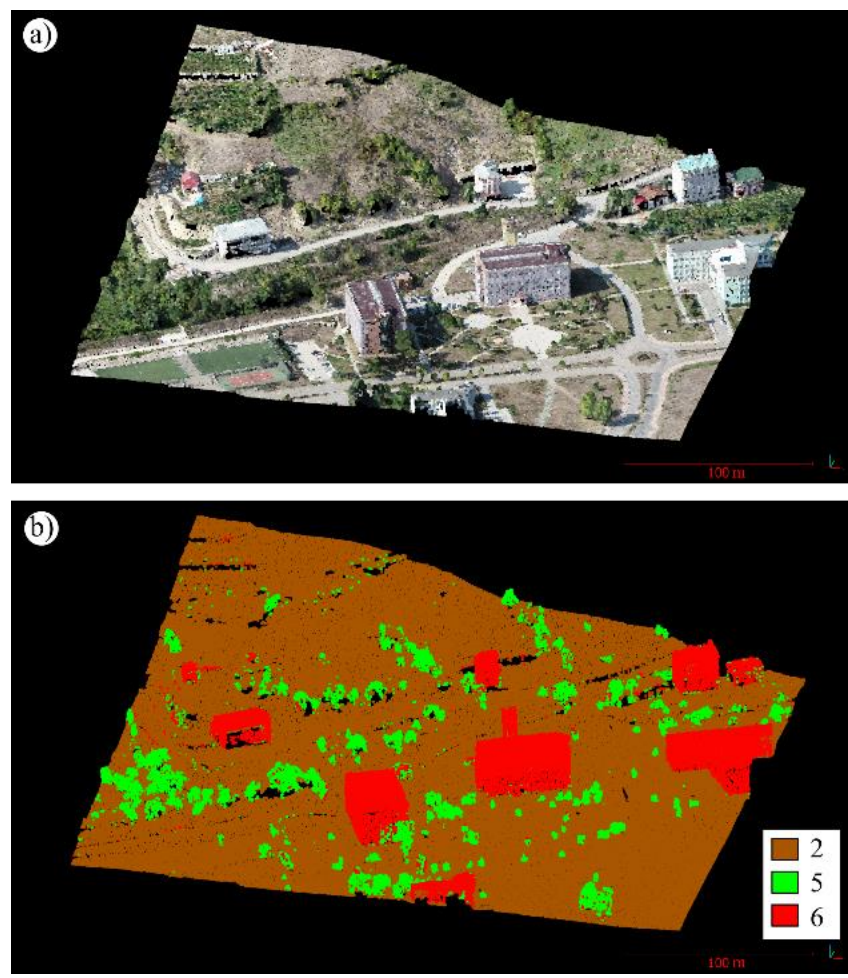

Figure 6. The classification obtained as a result of $\mathrm{RF}$ classifier, a) point clouds indicated by RGB bands, b) point clouds classified according to RF model (ground:2, vegetation:5 and building:6)

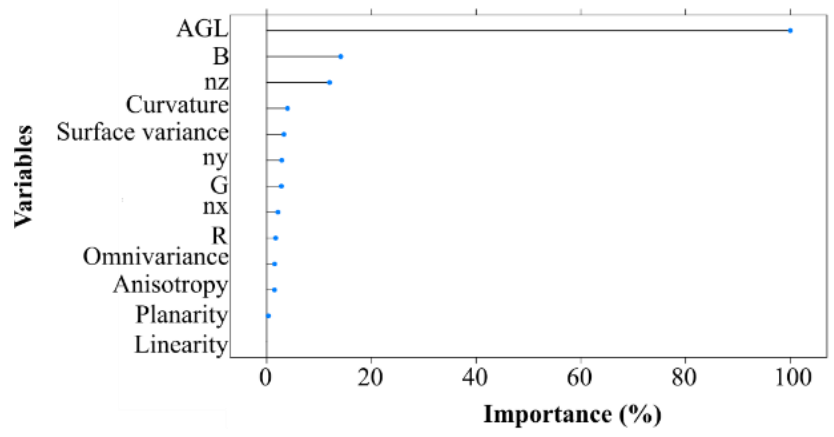

Figure 7. Importance ratios of covariance and radiometric properties evaluated in RF model

$\mathrm{RF}$ classifier has been performed to distinguish between three classes. A confusion matrix summarized the results of the RF classifier algorithm for further inspection. The confusion matrix are presented in Table 3. According to the confusion matrix, defective points are more likely in ground and vegetation classes. For the vegetation class, it is found that the $3 \mathrm{D}$ UAV points are not sufficiently detailed, due to they have a ground-like feature at the peaks of the trees and also the ground points could not be reconstructed from the leaves and branches of the trees as the main reasons for these errors. In addition, the presence of steep slopes prevents sharp curves between the ground and trees. In this 
context, tree points become the point feature that ensures the continuity of the ground points.

Statistical validation criteria on the test area are given in Table 4 regarding section 2.4. It is found that it is difficult to classify small plants especially close to the ground. Therefore, small vegetation points often complicated for 3D data. As a result, vegetation points in confusion matrices are classified as ground points and reduced the classification accuracy of other classes. The $\mathrm{RF}$ classification framework and obtained parameters of RF produce an overall accuracy of 0.96, and a Kappa coefficient of 0.91 .

During the classification, some of the common errors encountered the detection of planar points near the ground. Moreover, high points of the vegetation was classified as ground points, and the results of some decreases in accuracy values due to errors such as the scattering characteristic of on building facades. In order to avoid such errors and increase the accuracy values, it is necessary to reduce the noise points, to optimize the better neighbouring values expressing the surface characteristics and to improve the clustering analysis.

Table 3. Error matrix (confusion)

\begin{tabular}{|c|c|c|c|c|}
\hline & \multicolumn{4}{|c|}{ Reference data (Class) } \\
\hline (5) & & 2 & 5 & 6 \\
\hline$\underbrace{\frac{\pi}{U}}$ & 2 & 4269 & 93 & 45 \\
\hline$\underset{\frac{\pi}{0}}{\mathbb{\pi}}$ & 5 & 50 & 565 & 34 \\
\hline$\frac{0}{0}$ & 6 & 11 & 30 & 902 \\
\hline
\end{tabular}

Table 4. Classification statistical metrics

\begin{tabular}{lccc}
\hline & \multicolumn{3}{c}{ Class } \\
\cline { 2 - 4 } Sensitivity & 2 & 5 & 6 \\
\cline { 2 - 4 } Specificity & 0.9882 & 0.82887 & 0.9287 \\
PPV & 0.9311 & 0.98574 & 0.9908 \\
NPV & 0.9738 & 0.87994 & 0.9526 \\
Precision & 0.9682 & 0.97857 & 0.9859 \\
Recall & 0.9738 & 0.87994 & 0.9526 \\
F1 & 0.9882 & 0.82887 & 0.9287 \\
Prevalence & 0.9810 & 0.85364 & 0.9405 \\
Detection rate & 0.7220 & 0.11200 & 0.1660 \\
Detection prevalence & 0.7135 & 0.09283 & 0.1542 \\
Balanced accuracy & 0.7327 & 0.10550 & 0.1618 \\
\hline
\end{tabular}

When investigating the error source, it is significant whether the errors are occurred inly model-based or irregularities in point clouds and systematic errors. While the geometric properties of dense point clouds are detected in certain areas of measurement errors, it is almost impossible to detect for irregular objects (trees, vegetation near the ground etc.). Different studies are required to examine and eliminate these details. These errors in this study have not been studied.

Studies on the classification of point clouds can be applied to increase RF model classifier performances and accuracy criteria in parallel with the robust algorithms developed in future periods. Robust methods can be developed that are more optimized for geometric features and are not affected by the density changes of the points. However, some confusion computation stages and time may increase in the calculated point features.
For this, optimization and hardware requirements may increase. Different results are produced according to different studies and examples (Sevgen 2019; Nevalainen et al. 2017). Overall accuracy value is obtained as $96 \%$ in this study. For instance, when looking at the accuracy criteria in other studies, the accuracy values obtained are close and high accuracy. Kim and Sohn (2012) achieved a result of the experiment, 93.9\% with RF model.

$\mathrm{R}$ codes containing the methods applied in this article can be examined and used freely from Zeybek 2020.

\section{CONCLUSION}

The random forest machine learning algorithm has a comprehensive adaptability in the industry and in many research studies. Decision tree-based designs make it easier to interpret the model and the result, while intuitively approaching actual classification problems, increasing the correct estimation rate. To compare the proposed methodology directly with different methods, point clouds of similar structure and application parameters are needed. However, the adequacy of the model can be tested more accurately by applying the generated RF model of the obtained point clouds from different areas.

It has been shown in this study that UAV based dense point clouds can be classified according to their geometric characteristics and radiometric features. Although high accuracy values are obtained, there may still be incorrect classification points in some areas of the data. Therefore, it is necessary to improve this classification data with the help of operators after automatic classification. Further studies should be conducted to increase the accuracy model and improve its performance with robust feature estimation.

\section{ACKNOWLEDMENT}

We would like to acknowledge the financial support of Artvin Coruh University Scientific Research Projects Coordinatorship for the project number "2019.F40.02.02"

\section{REFERENCES}

Akar Ö \& Güngör O (2012). Classification of multispectral images using Random Forest algorithm. Journal of Geodesy and Geoinformation, 1(2), 105-112. DOI: 10.9733/jgg.241212.1

Akgül M, Yurtseven H, Demir M, Akay A E, Gülci S \& Öztürk T (2016). Usage opportunities of generating digital elevation model with unmanned aerial vehicles on forestry. Journal of the Faculty of Forestry Istanbul University, 66(1), 104-118 DOI:10.17099/jffiu.23976 (in Turkish)

Arya S, Mount D, Kemp S E \& Jefferis G (2019). RANN: Fast nearest neighbour search (wraps ANN library) using 12 metric. R package version 2.6, 1. Retrieved from: https://rdrr.io/cran/RANN/

ASPRS (2019). LAS Specification 1.4 - R14. American Society for Photogrammetry and Remote Sensing. 
Retrieved from http://www.asprs.org/wpcontent/uploads/2019/03/LAS_1_4_r14.pdf

Bivand R S, Pebesma E \& Gomez-Rubio V (2008). Applied spatial data analysis with R. ISBN: 978-1-46147618-4, Springer, New York.

Blomley R, Weinmann M, Leitloff J \& Jutzi B (2014). Shape distribution features for point cloud analysis - A geometric histogram approach on multiple scales. ISPRS Annals of Photogrammetry, Remote Sensing and Spatial Information Sciences, II-3, 9-16. DOI: 10.5194/isprsannals-II-3-9-2014

Breiman L (2001). Random forests. Machine learning, 45(1), 5-32.

Chen B, Shi S, Gong W, Zhang Q, Yang J, Du L, Sun J, Zhang Z \& Song S (2017). Multispectral liDAR point cloud classification: A two-Step approach. Remote Sensing, 9(4), 373. DOI: 10.3390/rs9040373

CloudCompare. (2013). Telecom ParisTech (version 2.4) [GPL software]. EDF R\&D. Retrieved from http://www.danielgm.net/cc/

Cutler D R, Edwards Jr T C, Beard K H, Cutler A, Hess K T, Gibson J \& Lawler J J (2007). Random forests for classification in ecology. Ecology, 88(11), 27832792. DOI:10.1890/07-0539.1

Çetinkaya B \& Toz G (2007). Usage of error matrix in the accuracy assessment of geographic data selection results. İTÜDERGİİ/d, 6(5-6), 59-68. (in Turkish)

Çömert R, Matci D K \& Avdan, U. (2019). Object based burned area mapping with random forest algorithm. International Journal of Engineering and Geosciences, 4(2), 78-87. DOI:10.26833/ijeg.455595

de Almeida C T, Galvao L S, Aragao L E D E, Ometto J P H B, Jacon A D, Pereira F R D, et al. (2019). Combining LiDAR and hyperspectral data for aboveground biomass modeling in the Brazilian Amazon using different regression algorithms. Remote Sensing of Environment, 232. DOI:10.1016/j.rse.2019.111323

Demir N (2015). Various methods to detect buildings using image and lidar data. Havacıllk ve Uzay Teknolojileri Dergisi, 8(1), 55-65. (in Turkish)

Guo L, Chehata N, Mallet C \& Boukir S (2011). Relevance of airborne lidar and multispectral image data for urban scene classification using Random Forests. ISPRS Journal of Photogrammetry and Remote Sensing, 66(1), 56-66. doi:10.1016/j.isprsjprs.2010.08.007

Guyot A, Lennon M, Thomas N, Gueguen S, Petit T, Lorho $\mathrm{T}$, Cassen S \& Hubert-Moy L (2019). Airborne hyperspectral imaging for submerged archaeological mapping in shallow water environments. Remote Sensing, 11(19). DOI: $10.3390 /$ rs11192237

Hackel T, Wegner J D \& Schindler K (2017). Joint classification and contour extraction of large 3D point clouds. ISPRS Journal of Photogrammetry and Remote Sensing, 130, 231-245. DOI:10.1016/j.isprsjprs.2017.05.012

Karakaş G (2018). An approach for building extraction using lidar point cloud data and high resolution orthophotos. Master's Thesis, Hacettepe University, Ankara (in Turkish).
Kashani A G, Olsen M J, Parrish C E \& Wilson N (2015). A review of LIDAR radiometric processing: From $\mathrm{Ad}$ Hoc intensity correction to rigorous radiometric calibration. Sensors, 15(11), 28099-28128. DOI: $10.3390 / \mathrm{s} 151128099$

Kim H B \& Sohn G (2012). Random forests based multiple classifier system for power-Line scene classification. International Archives of the Photogrammetry, Remote Sensing and Spatial Information Sciences, 38(5), W12, 253-258. DOI: 10.5194/isprsarchivesXXXVIII-5-W12-253-2011

Kraus K \& Pfeifer N (1998). Determination of terrain models in wooded areas with airborne laser scanner data. ISPRS Journal of Photogrammetry and Remote Sensing, 53(4), 193-203. DOI: 10.1016/S09242716(98)00009-4

Kuhn M (2008). Building predictive models in R using the caret package. Journal of Statistical Software, 28(5), 1-26.

Liaw A \& Wiener M (2002). Classification and regression by randomForest. R news, 2(3), 18-22.

Lowe D G (2004). Distinctive image features from ScaleInvariant keypoints. International Journal of Computer Vision, 60(2), 91-110. DOI:10.1023/b:Visi.0000029664.99615.94

Luo L, Wang X, Guo H, Lasaponara R, Zong X, Masini N, et al. (2019). Airborne and spaceborne remote sensing for archaeological and cultural heritage applications: A review of the century (1907-2017). Remote Sensing of Environment, 232. DOI:10.1016/j.rse.2019.111280

Nevalainen O, Honkavaara E, Tuominen S, Viljanen N, Hakala T. et al. (2017). Individual tree detection and classification with UAV-Based photogrammetric point clouds and hyperspectral imaging. Remote Sensing, 9(3). DOI: 10.3390/rs9030185

Niemeyer J, Rottensteiner F \& Soergel U (2014). Contextual classification of lidar data and building object detection in urban areas. ISPRS Journal of Photogrammetry and Remote Sensing, 87, 152-165. doi:10.1016/j.isprsjprs.2013.11.001

Niu Z, Xu Z, Sun G, Huang W, Wang L, Feng M, Li W, He W, Gao S (2015). Design of a new multispectral waveform LiDAR instrument to monitor vegetation. IEEE Geoscience and Remote Sensing Letters, 12(7), 1506-1510. DOI: 10.1109/LGRS.2015.2410788

Ok A Ö, Akar Ö \& Güngör O (2011). Classification of crops in agricultural lands using random forest classification method. TUFUAB VI. Teknik Sempozyumu, Antalya, Turkey (in Turkish).

Özbay E \& Çınar A (2016). A metrical approach to classification of the object modelling with point cloud data. Afyon Kocatepe University Journal of Science and Engineering, 16,128 - 136 (in Turkish)

Özdemir E \& Remondino F (2019) Classification of aerial point clouds with deep learning. International Archives of the Photogrammetry, Remote Sensing and Spatial Information Sciences, 42(2), 103-110. DOI: 10.5194/isprs-archives-XLII-2-W13-103-2019

Öztürk O, Bilgilioğlu B B, Çelik M F, Bilgilioğlu S S \& Uluğ $R$ (2017). The investigation of the height and the camera angle in the production of orthoimage with 
images of unmanned aerial vehicle (UAV). Geomatik, 2(3), 135-142. DOI:10.29128/geomatik.327049 (in Turkish).

Pan Y, Zhang X, Cervone G \& Yang L (2018). Detection of asphalt pavement potholes and cracks based on the unmanned aerial vehicle multispectral imagery. IEEE Journal of Selected Topics in Applied Earth Observations and Remote Sensing, 11(10), 37013712. DOI: 10.1109/JSTARS.2018.2865528

Pandey P C, Koutsias N, Petropoulos G P, Srivastava P K \& Dor E B (2019). Land use/land cover in view of earth observation: data sources, input dimensions, and classifiers-a review of the state of the art. Geocarto International. DOI:10.1080/10106049.2019.1629647

Ramasubramanian K \& Singh A (2017). Machine learning using R: Springer, Berkeley. ISBN: 978-1-48424215-5

Roussel J-R \& Auty D (2017). lidR: Airborne LiDAR data manipulation and visualization for forestry applications. Retrieved from:https://github.com/Jean-Romain/lidR.

Sevgen S C (2019). Airborne lidar data classification in complex urban area using random forest: A case study of Bergama, Turkey. International Journal of Engineering and Geosciences, 4(1), 45-51. DOI:10.26833/ijeg.440828

Shan J \& Toth C K (2018). Topographic Laser Ranging and Scanning. Taylor \& Francis Group. ISBN:13-978-14987-7227-3

Sohn G, Jwa Y \& Kim H B (2012). Automatic powerline scene classification and reconstruction using airborne lidar data. ISPRS Annals of Photogrammetry, Remote Sensing and Spatial Information Sciences, I-3, 167-172. Doi: 10.5194/isprsannals-I-3-167-2012

Şahin B, Güzel C, Baş S \& Türker M (2018). 3DETECTORLIDAR nokta bulutu verisinden otomatik ağaç konumu belirleme sistemi. VII. Uzaktan AlglamaCBS Sempozyumu, Eskisehir, 21 (in Turkish).

Taşcı A E \& Onan A (2016). K-en yakın komşu algoritması parametrelerinin sınıflandırma performansı üzerine etkisinin incelenmesi. Akademik Bilişim (in Turkish).

Team R C (2019). R: A Language and Environment for Statistical Computing In R Foundation for Statistical Computing, Vienna, Austria.

Tóvári D \& Pfeifer N (2005). Segmentation based robust interpolation-a new approach to laser data filtering. International Archives of Photogrammetry, Remote Sensing and Spatial Information Sciences, 36(3/19), 79-84.

Ulvi A (2018). Analysis of the utility of the unmanned aerial vehicle (UAV) in volume calculation by using photogrammetric techniques. International Journal of Engineering and Geosciences. 3(2), 43-49. DOI:10.26833/ijeg.377080

Ulvi A \& Toprak A S (2016). Investigation of threedimensional modelling availability taken photograph of the unmanned aerial vehicle: Sample of Kanlidivane Church. International Journal of Engineering and Geosciences, 1(1), 1-7. DOI: 10.26833/ijeg.285216

Ulvi A, Yakar M, Yiğit A Y \& Kaya Y (2020). Production of 3 Dimensional Point Clouds and Models of Aksaray Kızıl Kilise by Using UAVs and Photogrammetric Techniques. Geomatik, 5(1), 19-26. DOI:10.29128/geomatik.560179 (in Turkish)

Vosselman G (2000). Slope based filtering of laser altimetry data. International Archives of Photogrammetry and Remote Sensing, 33, 935-942.

Wei G, Shalei S, Bo Z, Shuo S, Faquan L, Xuewu C (2012). Multi-wavelength canopy LiDAR for remote sensing of vegetation: Design and system performance. ISPRS Journal of Photogrammetry and Remote Sensing, 69 , 1-9. DOI:10.1016/j.isprsjprs.2012.02.001

Wichmann V, Bremer M, Lindenberger J, Rutzinger M, Georges C, Petrini-Monteferri F (2015). Evaluating the potential of multispectral airborne lidar for topographic mapping and land cover classification. ISPRS Annals of the Photogrammetry, Remote Sensing and Spatial Information Sciences, 2(3)(W5), 113-119. DOI:10.5194/isprsannals-II-3-W5-1132015

Yadav M \& Singh A K (2017). Rural road surface extraction using mobile LiDAR point cloud data. Journal of the Indian Society of Remote Sensing, 46,531-538. DOI: 10.1007/s12524-017-0732-4

Zeybek M \& Şanlığlu İ (2019a). Point cloud filtering on UAV based point cloud. Measurement, 133, 99-111. DOI:10.1016/j.measurement.2018.10.013

Zeybek M \& Şanlıoğlu İ (2019b). A study on determination of topographical surface changes by image processing techniques. Journal of Natural Hazards and Environment, 5(2), 350-367. DOI: 10.21324/dacd.531719

Zeybek M (2020) PCL-RandomForest-Classification Retrieved from: https://github.com/mzeybek583/PCLRandomForest-Classification.

Zhang K, Chen S C, Whitman D, Shyu M L, Yan J, Zhang C (2003). A progressive morphological filter for removing nonground measurements from airborne LIDAR data. IEEE Transactions on Geoscience and Remote Sensing, 41(4), 872-882. DOI:10.1109/TGRS.2003.810682

Zhang W, Qi J, Wan P, Wang H, Xie D, Wang X, Yan G (2016). An Easy-to-Use airborne liDAR data filtering method based on cloth simulation. Remote Sensing, 8(6), 501. DOI: $10.3390 /$ rs8060501

Zou X, Cheng M, Wang C, Xia Y \& Li J (2017). Tree classification in complex forest point clouds based on deep learning. IEEE Geoscience and Remote Sensing Letters, 14(12), 2360-2364. DOI: $10.1109 /$ LGRS.2017.2764938 